\title{
BMJ Open Remote mental health services: a mixed- methods survey and interview study on the use, value, benefits and challenges of a national video consulting service in NHS Wales, UK
}

\author{
Gemma Johns (1) , ${ }^{1}$ Anna Burhouse, ${ }^{2}$ Jacinta Tan, ${ }^{3}$ Oliver John, ${ }^{4}$ Sara Khalil, \\ Jessica Williams, ${ }^{1}$ Bethan Whistance, ${ }^{1}$ Mike Ogonovsky, ${ }^{1}$ Alka Ahuja (D) ${ }^{1}$
}

To cite: Johns G, Burhouse A, Tan J, et al. Remote mental health services: a mixedmethods survey and interview study on the use, value, benefits and challenges of a national video consulting service in NHS Wales, UK. BMJ Open 2021;11:e053014. doi:10.1136/ bmjopen-2021-053014

- Prepublication history and additional supplemental materia for this paper are available online. To view these files, please visit the journal online (http://dx.doi.org/10.1136/ bmjopen-2021-053014).

Received 01 May 2021 Accepted 27 August 2021

\section{Check for updates}

(c) Author(s) (or their employer(s)) 2021. Re-use permitted under CC BY-NC. No commercial re-use. See rights and permissions. Published by BMJ.

${ }^{1}$ Department of Informatics, TEC Cymru, Aneurin Bevan University Health Board, Gwent, UK ${ }^{2}$ Northumbria Healthcare NHS Foundation Trust, Northumbria, UK

${ }^{3}$ Child \& Adolescent Mental Health Services, Aneurin Bevan University Health Board, Ty Bryn St Cadocs Hospital, Newport, UK ${ }^{4}$ Royal College of Psychiatrists Wales, Cardiff, UK

Correspondence to

Gemma Johns;

gemma.johns3@wales.nhs.uk

\section{ABSTRACT}

Social distancing laws during the first year of the pandemic, and its unprecedented changes to the National Health Service (NHS) forced a large majority of services, especially mental health teams to deliver patient care remotely. For many, this approach was adopted out of necessity, rather than choice, thus presenting a true 'testing ground' for remote healthcare and a robust evaluation on a national and representative level. Objective To extract and analyse mental health specific data from a national dataset for 1 year (March 2020 March 2021).

Design A mixed-methods study using surveys and interviews.

Setting In NHS mental health services in Wales, UK. Participants With NHS patients and clinicians across child and adolescent, adult and older adult mental health services.

Outcome measures Mixed methods data captured measures on use, value, benefits and challenges of video consulting (VC).

Results A total of 3561 participants provided mental health specific data. These data and its findings demonstrate that remote mental health service delivery, via the method of VC is highly satisfactory, well-accepted and clinically suitable for many patients, and provides a range of benefits to NHS patients and clinicians. Interestingly, clinicians working from 'home' rated VC more positively compared with those at their 'clinical base'. Conclusions Post 1-year adoption, remote mental health services in Wales UK have demonstrated that VC is possible from both a technical and behavioural standpoint. Moving forward, we suggest clinical leaders and government support to sustain this approach 'by default' as an option for NHS appointments.

\section{BACKGROUND}

The pandemic resulted in a paradigm shift in the provision of mental health services due to mandatory social distancing laws. ${ }^{12}$ From March 2020, the UK, along with the National Health Service (NHS) observed a significant

\section{STRENGTHS AND LIMITATIONS OF THIS STUDY}

$\Rightarrow$ This is a mixed-methods study exploring the remote delivery of mental health services across all Health Boards within one UK nation-Wales.

$\Rightarrow$ COVID-19 presented an opportunity within National Health Service (NHS) Wales to implement and evaluate video consulting in large numbers, across a wide range of NHS services.

$\Rightarrow$ This study provides both a patient and clinician comparison and a comparison across child and adolescent, adult and older adult mental health services which adds value to the current literature.

$\Rightarrow$ Due to COVID-19 restrictions, the study of video consulting has been limited to survey and interview methodologies. However, as restrictions lift, measures around efficacy and effectiveness are now underway, this paper acts as a basis for the new evaluation and research currently being conducted in this area.

$\Rightarrow$ Limitations of this study include small sample size in older adult patients; limited demographic participant information; and that the survey data are likely to be from some recurring patients, thus potentially skewing the sample.

decrease in access to face-to-face appointments, and as a result, an increase in remote services. ${ }^{12}$ One common remote method for conducting appointments with patients was the use of video consulting (VC). ${ }^{3}$ VC can be comparable to face-to-face contact, ${ }^{4}$ and in some instances, a preferred method to face to face. ${ }^{5} \mathrm{VC}$ within mental health services has been internationally utilised for decades, yet the unprecedented circumstances of the pandemic brought to light its widespread ability, use, value, benefits and challenges to countries such as the UK. ${ }^{12}$ The use of $\mathrm{VC}$ permits services to continue across a wide range of mental healthcare conditions, appointment types, sociodemographic groups 
and health condition status. ${ }^{6-10}$ Furthermore, it is sometimes considered more suitable for reaching underserved and isolated populations. ${ }^{2} \mathrm{VC}$ is reported to provide quality ensured, yet cost-effective mental health support, care and treatment, ${ }^{11}$ while reducing patient waiting times and the likelihood of Did Not Attends (DNAs), number of admissions and lengths of stay in hospitals-ultimately relieving pressure on NHS staff and services. ${ }^{12}$ However, there is often some concerns expressed regarding the use of $\mathrm{VC}$ services within mental health, as it is felt that rapport may not be effectively built, or there is a larger risk of missing out on certain cues or clinical signs, compared with face-to-face appointments. ${ }^{147}$

\section{AIMS}

The aim of this paper is to present 1 year of remote mental health service delivery data as an evidence base from a UK Wales national and representative sample, using mixed methodology datasets. This paper will discuss findings on the use, value, benefits and challenges of remote services via the method of VC, which is based on the perspective and experience of patients and clinicians across a range of child and adolescent mental health services (CAMHS), adult and older adult mental health services. The findings will be discussed by comparing CAMHS and adult mental health service in dataset 1 , comparing patient and clinician feedback in dataset 2 . This is combined in dataset 3 .

\section{METHODS}

\section{Design, setting, participants}

This paper is a mixed-methods study, which combines findings from three datasets from the NHS Wales VC Service. ${ }^{13} 14$ This includes survey and interview data captured across the first 1year of its national adoption (March 2020 to March 2021). Technology Enabled Care (TEC) Cymru are an NHS Wales multidisciplinary team, with clinical, research, programme and technical expertise. TEC Cymru are an AllWales digital service, that enable the sustainable use, scale up and spread of value added technology. ${ }^{13}$ The NHS Wales VC Service is one of TEC Cymru's programmes that was rolled out nationally prior to the COVID-19 pandemic, ${ }^{14}$ and made available to all Health Boards and Trusts in Wales. The data in this paper is the All-Wales data captured across all 7 Health Boards, across and a range of NHS Wales mental health settings and conditions (see online supplemental appendix 1 for full list). Participant eligibility included NHS Wales mental health patients across age groups, and NHS Wales mental health clinicians using the VC Service during the 1 year of adoption.

\section{Sampling and recruitment}

The sampling approach used for surveys and interviews was opportunity sampling, due to access of the VC intervention and ability to attach a survey link, and access to $\mathrm{VC}$ user emails to contact for an interview. There is risk of some bias of those willingly completing the surveys or taking part in interviews as potentially more 'positive' or more 'negative' towards the VC intervention, potentially missing out more 'neutral' responses. Therefore, additional efforts were taken to reduce this bias, and snowballing sampling was also explored via adverts on social media platforms (@teccymru) and through other personal or professional networks for additional interviews.

The surveys appeared as internet browser pop-ups at the end of each VC appointment-one per clinician and patient, and were completed immediately as live data to reflect the use, value, benefits and challenges of VC. A copy of the surveys can be found as online supplemental appendix 1). The interviewees were recruited in the course of filling in the post-VC surveys, participants were invited to fill in their contact details if they were willing to be interviewed and this was subsequently carried out by a research assistant, or were contacted directly by the TEC Cymru team via an email invite.

\section{Sample size}

A total of 3561 participants were extracted from the NHS Wales VC Service national dataset as representing mental health data. This mental health data subsample is discussed in this paper. Based on the total number of mental health VC appointments conducted during this time $(n=7350)$, the response rate is almost $50 \%$. The data include sections from the following three datasets.

\section{Survey 1 data}

Measures on 'use and value'; date period: March-August 2020; total mental health respondents $(\mathrm{N}=1027)$.

\section{Survey 2 data}

Measures on 'benefits and challenges'; date period: September 2020-March 2021; total mental health respondents $(\mathrm{N}=2453)$.

\section{Follow-up interviews}

Themes on 'use, value, benefits and challenges'; date period: June to December 2020; total mental health respondents $(\mathrm{N}=81)$.

\section{Recruitment and approvals}

Full consent was obtained from all participants. At the end of each survey, a statement of consent and compulsory tick box was required. At each interview, full consent was verbally obtained. TEC Cymru obtained full service evaluation approval and risk assessments for all evaluation conducted in associated to the use of the NHS Wales VC Service.

\section{Measures}

In survey 1, respondents were asked questions to capture measures on 'use and value' of VC. There were six questions: quality rating of VC; prevention of the need for face-to-face contact; patient demographics; patients' usage; clinicians work/home location and type of appointment. For analysis of this dataset, the data were split between CAMHS ( $\mathrm{n}=87$ patient responses and $\mathrm{n}=161$ clinician responses) and adult services, $(\mathrm{n}=298$ patient responses and $\mathrm{n}=481$ clinician responses) in 
order to compare and contrast. In survey 2, respondents were asked questions to capture measures on 'benefits and challenges' when using VC. This data are presented as a comparison between patients and clinicians. For this dataset, there were 1563 patients (CAMHS 156; Adult 1383) and 890 clinicians. Unlike survey 1 , these data were discussed in comparison of patient and clinician perspectives only, rather than comparing and contrasting across services, due to differences in sample sizes across CAMHS and adult services. Please note that each response in surveys was counted rather than the number of people; a person actively using VC for multiple appointments and filling in the survey after each encounter could be counted more than once. The final section of the paper presents a narrative overview of the experience of $\mathrm{VC}$ in mental health services which is broken down into thematic categories, which includes data from interviews and free text narrative captured in surveys.

\section{Statistical methods}

Survey 1 data were analysed in terms of distributions of responses, as well as differences that exist between specific groups of respondents (patients and clinicians). Statistical tests of differences were conducted to compare groups on the quality ratings of VC. The quality rating was measured ordinally, as set responses were given for respondents to select. Mann-Whitney $\mathrm{U}$ tests were carried out to test the statistical differences between two groups of respondents. Survey 2 data were analysed using descriptive methods of percentages and matched with qualitative data that was manually extracted and thematically analysed to explore emerging themes.

\section{Patient and public involvement \\ No patient involved.}

\section{RESULTS}

\section{Participants and setting}

A total of 3561 participants were extracted from three NHS Wales VC Service national datasets as representing mental health data. Across all three datasets, the participants included both NHS Wales patients across age and gender groups and mental health conditions, and clinicians across a wide range of mental health service settings in Wales (see online supplemental appendix 1 for full list).

\section{Dataset 1}

Use and value of remote mental health services

In survey 1 , respondents were asked questions to capture measures on 'use and value' when using VC. These data are split between CAMHS ( $\mathrm{n}=87$ patient responses and $\mathrm{n}=161$ clinician responses) and adult services, $(\mathrm{n}=298$ patient responses and $\mathrm{n}=481$ clinician responses) to compare and contrast. Table 1 displays the patient age ranges.

\section{Patient demographics}

Only $17 \%$ of the overall total number of responses were from patients in CAMHS compared with $83 \%$ in adult and older age mental health services. There was also a larger percentage of females $(60 \%)$ compared with males (38\%; $2 \%$ preferred not to say). Due to small numbers from older adult services alone, the remaining sections combine all adult together, as there were no differences identified between groups.

\section{Quality rating}

Using a Likert scale ranging from 1-star ('poor') to 5-stars ('excellent') respondents rated the quality of the $\mathrm{VC}$, with a total of $82.3 \%$ rating it as 'excellent', 'very good' or 'good'. Considering patients and clinicians separately, patients were more positive in the quality ratings, when compared with clinicians (figure 1), and this difference was found to be significant by a Mann-Whitney $\mathrm{U}$ $(\mathrm{U}=102889.0, \mathrm{p}<0.001)$.

When comparing responses for CAMHS and Adult services, these were very similar in their quality ratings, and no significant differences were revealed by statistical testing ( $\mathrm{U}=93324.0, \mathrm{p}=0.649)$. 86.6\% CAMHS $(\mathrm{n}=246)$ and $81.0 \%$ of adult $(\mathrm{n}=773)$ respondents rated VC 'excellent', 'very good' or 'good'. The responses are displayed in figure 2.

\section{Face-to-face prevention}

Respondents were asked whether the VC prevented the need for a face-to-face appointment, with the response options 'yes' or 'no'. $87.8 \%$ respondents stated that faceto-face was prevented, which was a positive outcome, given the risks of attending a clinical setting during the COVID-19 pandemic. When comparing CAMHS and adult services, face-to-face prevention was also similar, $81.4 \%$ in CAMHS and $89.8 \%$ in adult services.

\section{Patient usage}

Table 2 displays the responses to whether patients had used VC before, how many times and whether they would use it again or after COVID-19 had passed. This reveals that over half of the patients in both CAMHS and Adult services had used VC prior to their

Table 1 Age ranges (\% and freq) in CAMHS adult and older adult services

\begin{tabular}{|c|c|c|c|c|c|c|}
\hline & \multicolumn{2}{|c|}{ Child and adolescent } & \multicolumn{3}{|l|}{ Adult } & \multirow{2}{*}{$\begin{array}{l}\text { Older adult } \\
>65\end{array}$} \\
\hline & $<12$ & $13-17$ & $18-24$ & $25-44$ & $45-64$ & \\
\hline Percentage & 4 & 13 & 6 & 41 & 32 & 5 \\
\hline
\end{tabular}

CAMHS, child and adolescent mental health services. 


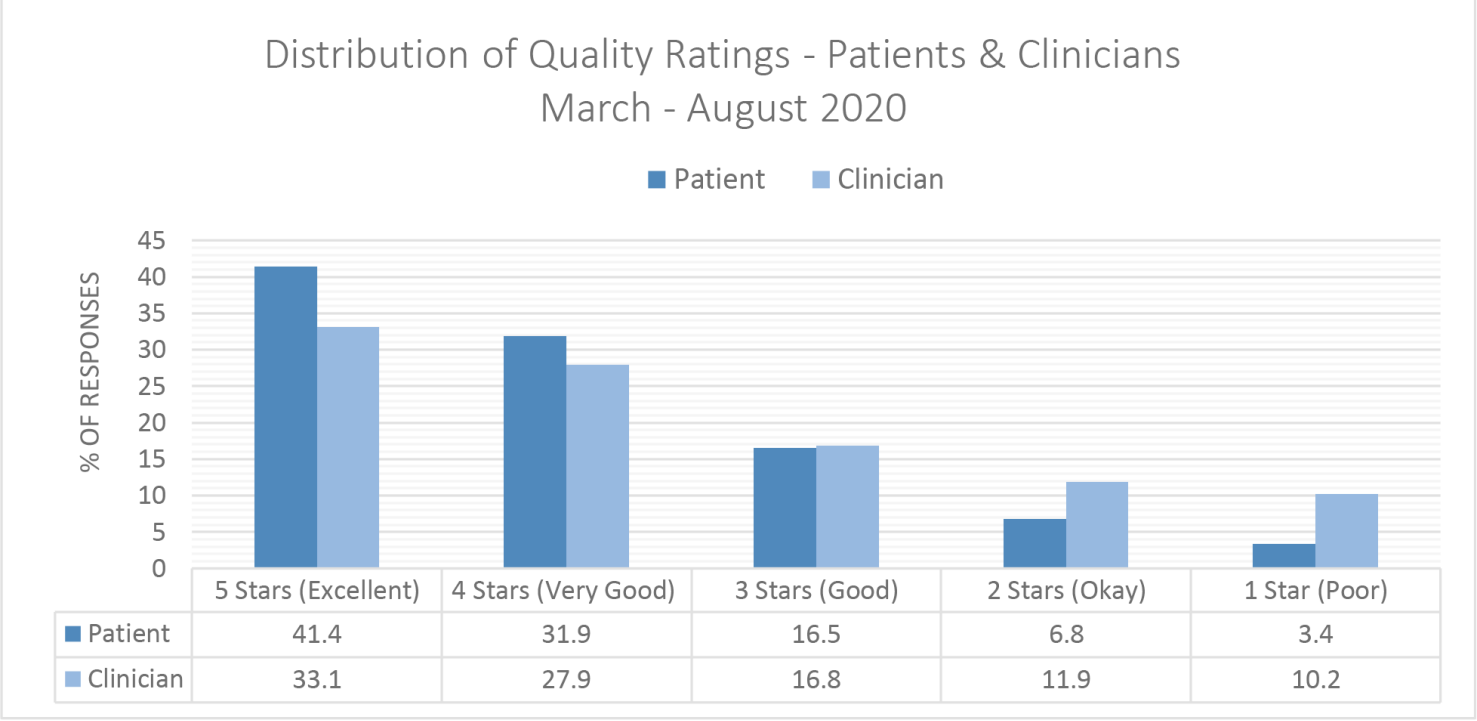

Figure 1 Distributions of responses for VC quality ratings by patients and clinicians. VC, video consulting.

appointment, with a higher proportion of patients having used it more often in Adult services previously. High percentages of respondents in both service types would consider using VC again.

Clinician home versus work location

A similar distribution of clinicians were working from their home and work location while using $\mathrm{VC}$, with $52 \%$ working from 'home' and 48\% from their 'work' (clinical base/office). Interestingly overall, those working from 'home' rated VC more positively than those working at their clinical base/office, revealed by a Mann-Whitney U test of difference, $\mathrm{U}=41899.5$, $\mathrm{p}=0.026$. Furthermore, clinical outcomes were not impacted by type of workplace, in that the prevention of face to face was comparable (85.2\% 'work'; $85.5 \%$ 'home'). Across both CAMHS and Adult services, there was a relatively even split between 'home' and 'work' bases, with slightly more $(53.1 \%)$ CAMHS clinicians (CC) based in 'work' and slightly more adult clinicians (AC) $(51.8 \%)$ based at 'home'. However, CC based at 'work' rated VC more positively than those working from 'home' ( $\mathrm{U}=2534.0, \mathrm{p}=0.04)$, whereas in adult services, 'home' workers rated VC more positively $(\mathrm{U}=20502.5, \mathrm{p}<0.001)$. These distributions are displayed in figure 3 .

\section{Appointment type}

The most common appointment type that was being conducted using VC was 'therapy' $(47.5 \%)$, followed by

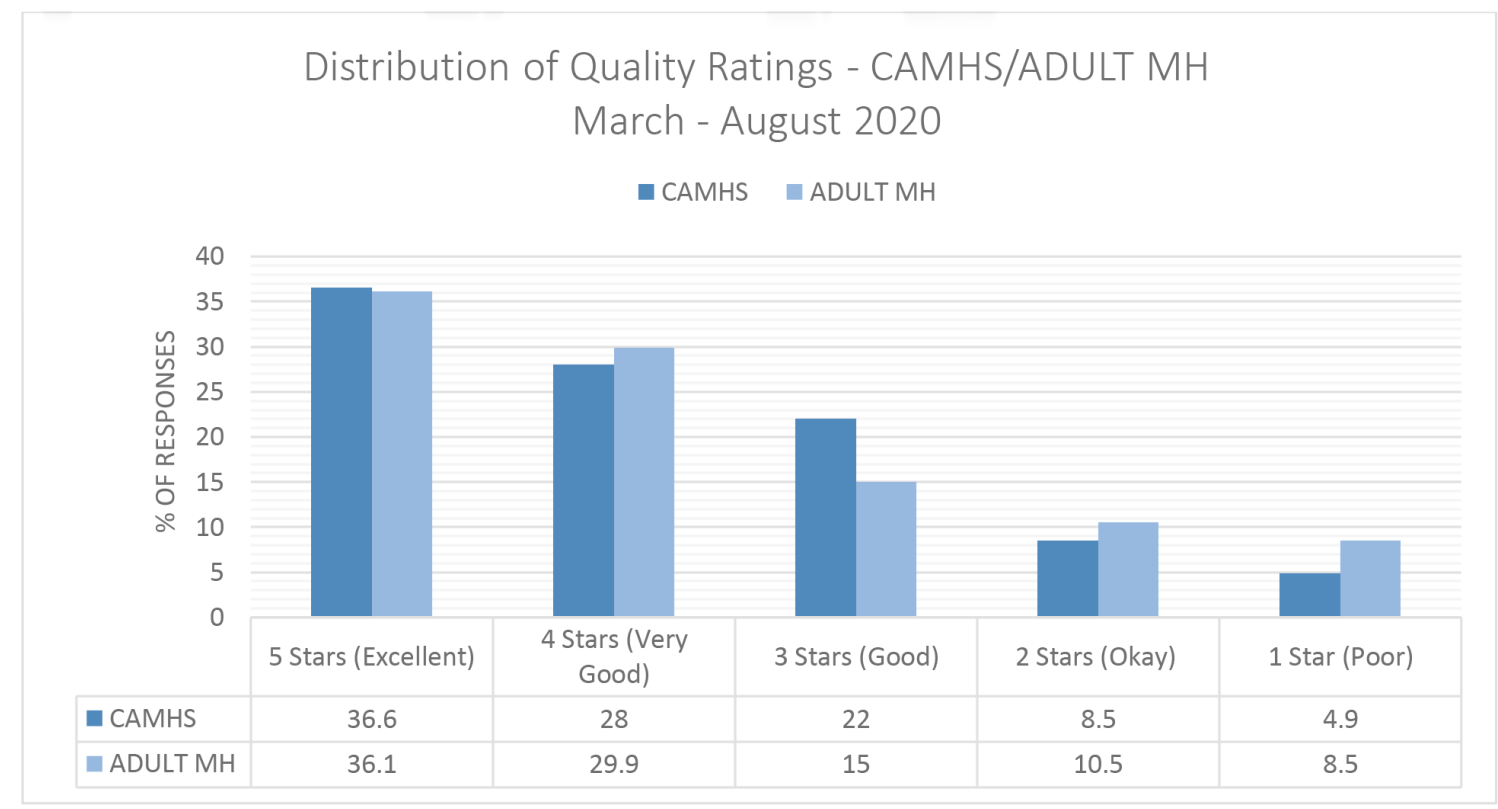

Figure 2 Distribution of responses for VC quality ratings by CAMHS and adult services. CAMHS, child and adolescent mental health services; $\mathrm{MH}$, mental health; $\mathrm{VC}$, video consulting. 
Table 2 Previous VC usage and preference on continued use

\begin{tabular}{lcc}
\hline & CAMHS & Adult services \\
\hline Used VC before? & & \\
\hline Yes & 54.9 & 52.4 \\
\hline No & 45.1 & 47.6 \\
\hline Freq. & 71 & 269 \\
\hline How many times? & & \\
\hline Once & 53.1 & 31.6 \\
\hline Twice & 12.2 & 16.9 \\
\hline Three or more & 34.7 & 51.4 \\
\hline Freq. & 49 & 177 \\
\hline Use again? & & 93.6 \\
\hline Yes & 91.2 & 6.4 \\
\hline No & 8.8 & 266 \\
\hline Freq. & 68 & \\
\hline $\begin{array}{l}\text { CAMHS, hild and adolescent mental health services; VC, video } \\
\text { consulting. }\end{array}$ &
\end{tabular}

'follow-up' (22.6\%) and 'first appointment' (13.8\%). Other types of appointments were reported considerably less, ranging from $0.5 \%$ (feedback/outcomes) to $4.1 \%$ (Advice) of total responses. Interestingly, face-to-face prevention was highest for 'therapy' (94.9\%) and 'first appointments' (96.6\%); compared with 'follow-ups' (89.1\%). The most common type of appointment in CAMHS was 'follow-up' $(31.1 \%)$, followed by 'therapy' (22.2\%). In adult services, the most common appointment was 'therapy' (54.1\%), followed by 'follow-ups' (20.3\%).

\section{Dataset 2}

Benefits and challenges of $\mathrm{VC}$

In survey 2, respondents were asked questions to capture measures on 'benefits and challenges' when using VC. These data are presented as a comparison between patients and clinicians. For this dataset, there were 1563 patients (CAMHS 156; Adult 1,383) and 890 clinicians. Figures 4 and 5 display the variation of benefits of $\mathrm{VC}$ and figures 6 and 7 display the challenges of $\mathrm{VC}$ rated by the patients and clinicians.

The highest rated benefits (based on scores between 'very beneficial' and 'beneficial') from the patient are 'lowered risk of infection' (89\%); 'no travel or parking', 'better for the environment' and being 'more convenient' (all 83\%). The lowest rated benefits were 'better family support' $(42 \%)$; and 'no time off work and school' (56\%) -but these two also rated the highest as non-applicable benefits.

The highest rated benefit (based on scores between 'very beneficial' and 'beneficial') from the clinicians is 'lowered risk of infection' (95\%); 'no travel or parking' $(86 \%)$ and 'more efficient use of clinical time/space' $(84 \%)$. Interestingly, the lowest rated benefits were benefits they perceived for the patient, including 'improved family support' (42\%); reduced likelihood of a DNA $(64 \%)$ and 'reduced waiting times' $(67 \%)$.

The highest rated challenge for the patients (based on scores 'a lot' or 'some') was for the 'preference of a face-toface appointment' (24\%). Interestingly, patients who stated a strong preference for face to face also rated all other benefits lower, suggesting that VC is more likely to be preferred 


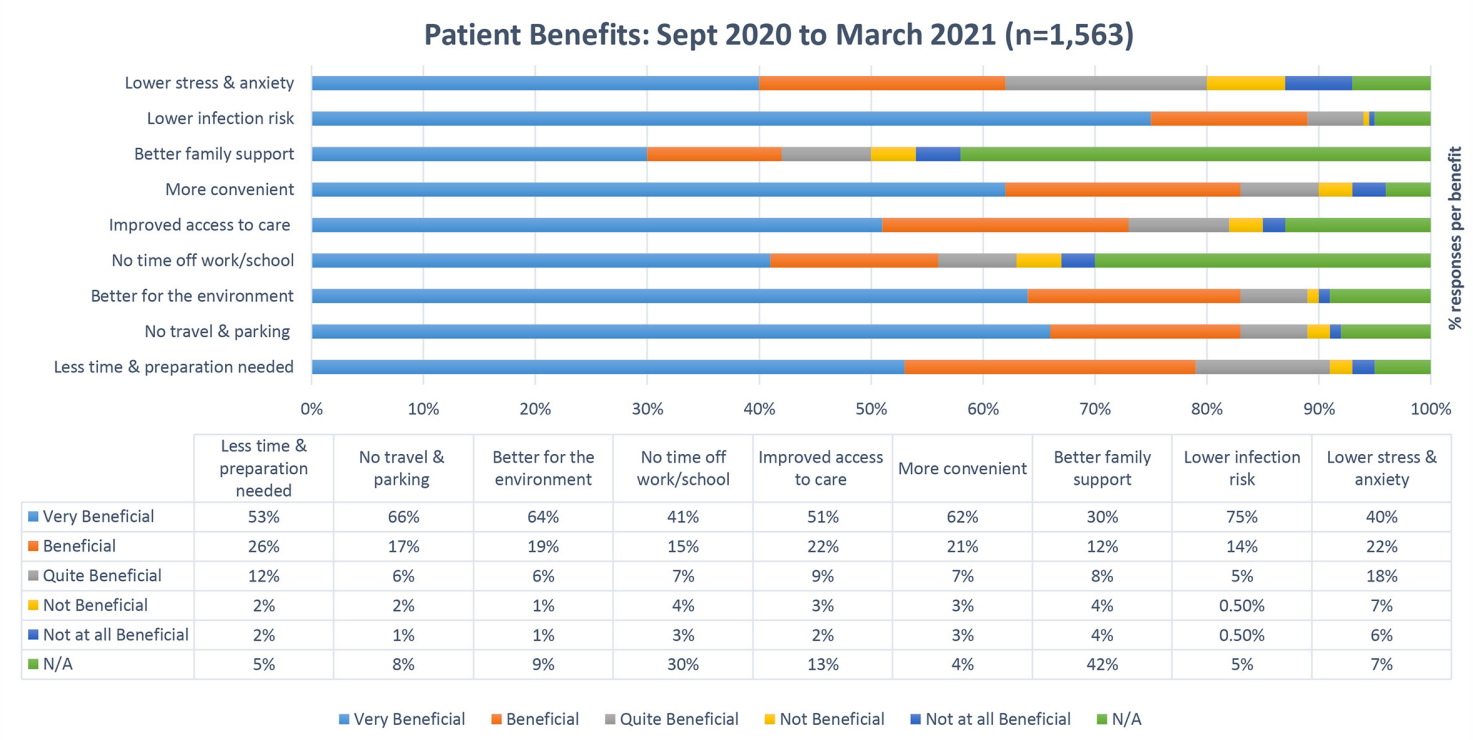

Figure 4 Percentage of patient benefits using VC. N/A, non-applicable; VC, video consulting.

by patients who stack other benefits higher, and therefore perceive the value of VC higher. The lowest rated challenge for patients were 'lack of privacy' (2\%) and 'unsuitability' $(3 \%)$.

The highest rated challenge for the clinicians (based on scores 'a lot' or 'some') was for poor 'audio/sound' $(26 \%)$ and 'video/picture' $(16 \%)$. The lowest rated challenge was 'clinicians lack of confidence' (1\%) and 'patient clinical suitability' (4\%).

\section{Travel saved and home working - patient and clinician}

Each patient was asked, by minutes and miles, how long would they have typically travelled to their appointment if attended in-person. Based on the survey 2 dataset (completed by 1469 patients during 1 September 2020 and 1 March 2021), many patients reported to have saved on travel, with a total of 1254 hours of travel saved, which on average suggests that a patient saves approximately $51 \mathrm{~min}$ per mental health NHS appointment (excluding trying to find parking). In addition, each clinician was asked if they are working from home, to calculate the average workforce location per day and how much travel they have saved. Based on the dataset (by 472 clinicians during 1 September 2020 and 1 March 2021), 53\% of clinicians reported to be working from home, and to have saved on travel, with a total of 608 hours of travel saved, which on average saves $77 \mathrm{~min}$ per clinician, per day in travel time (excluding trying to find parking).

\section{Clinician Benefits: Sept 2020 to March 2021 (n=890)}

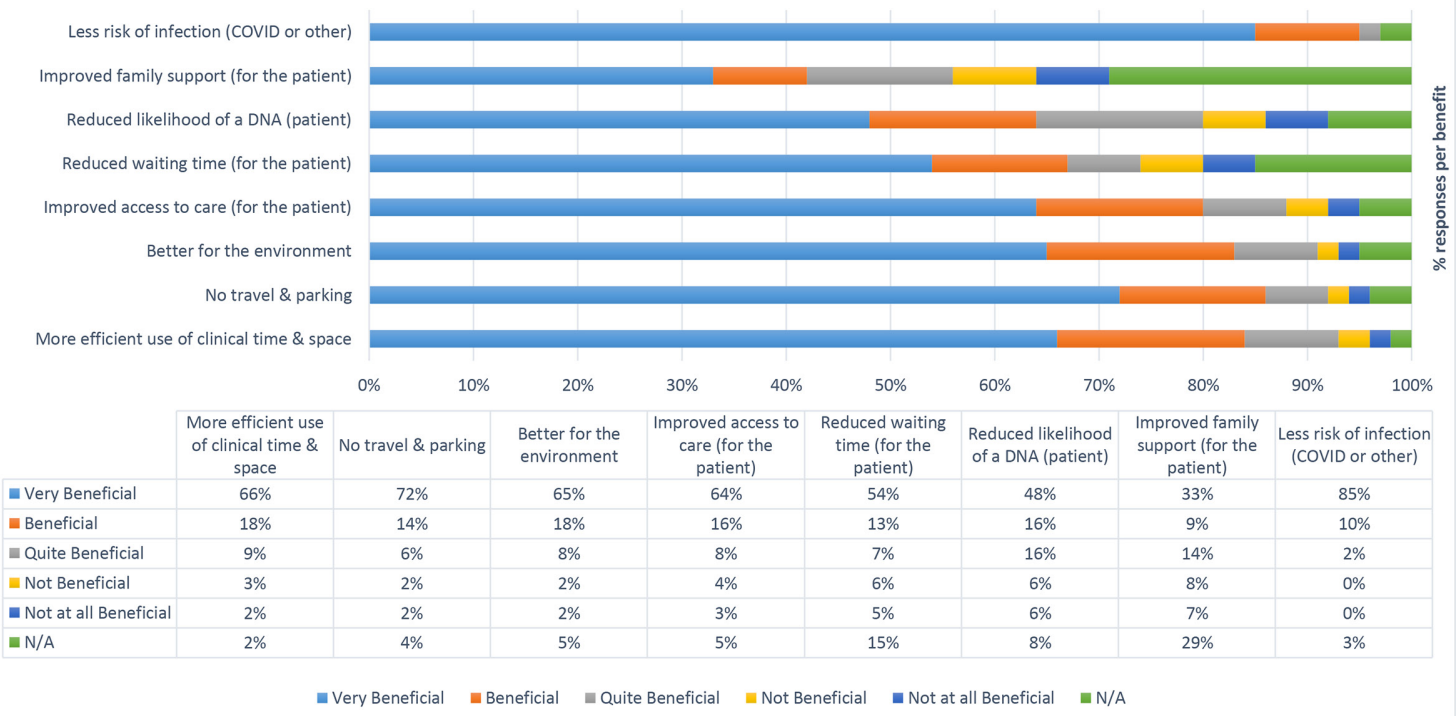

Figure 5 Percentage of clinicians benefits using VC. DNA, did not attend; N/A, non-applicable; VC, video consulting. 
Patient Reported Challenges - Sept 2020 to March 2021 ( $n=1,340)$

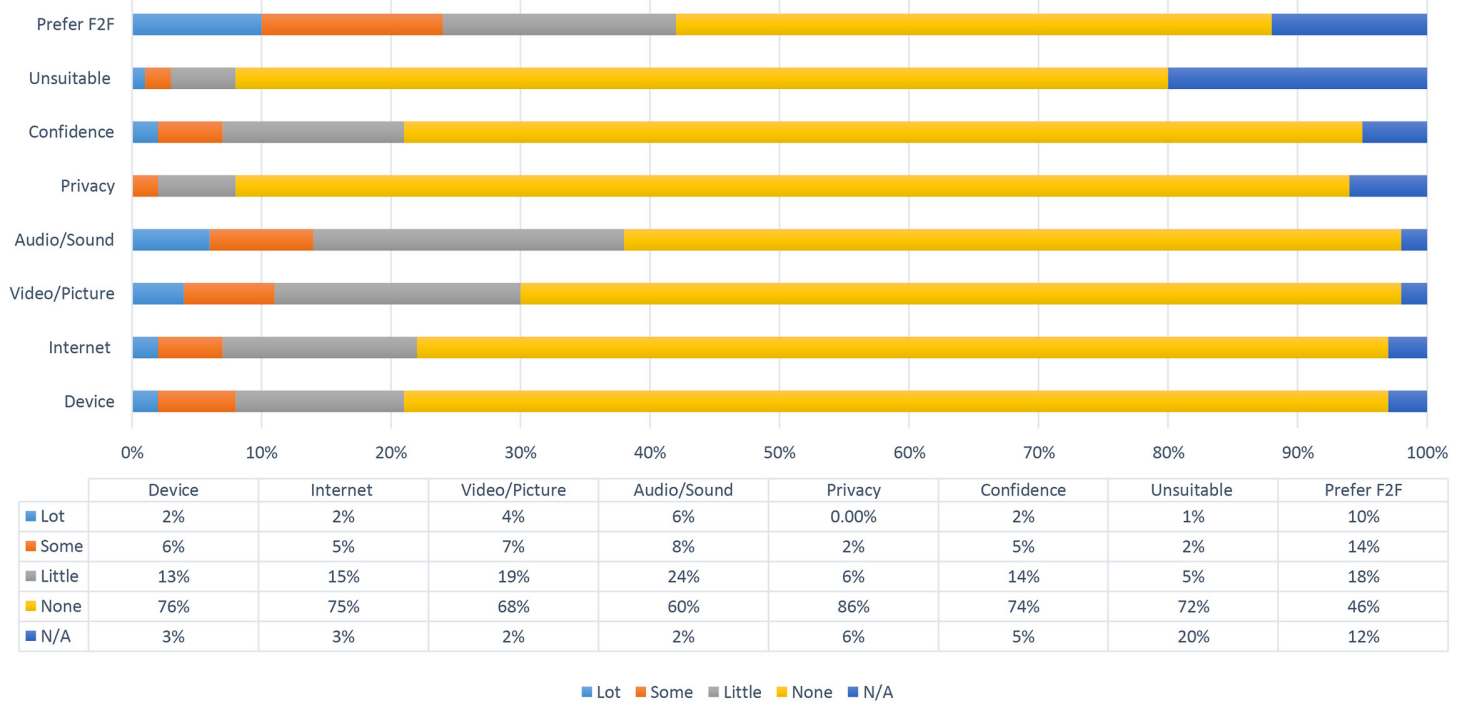

Figure 6 Distribution of patient challenges using VC. N/A, non-applicable; VC, video consulting.

\section{Dataset 3}

Narrative overview

This final section of the paper presents a narrative overview of the experience of $\mathrm{VC}$ in mental health services. For easier reading, the quotes and thematic categories are referenced as: CAMHS patient $(\mathrm{CP})$; adult patient (AP); older AP (OAP); CC; AC; older AC.

The mental health experience: perspective of patients

The use and 'preference' of VC in mental health services varies across patient groups and mental health conditions (see online supplemental appendix 1). For some patients, they 'still prefer face-to-face' $[\mathrm{AP}]$, feeling that ' $V C$ doesn't work well' for certain needs [AP] and that 'face-to-face is better, [because] video feels cut off' [CP]. An adult patient describes how '...the video supported me... but it is still quite a sterile environment and face-to-face would have more of a connection, for mental health at least' [AP]. Alternatively, an older age adult patient argues that 'face-to-face seems more personal, but it's probably more of a habit as the video was just as good' [OAP]. For others across patient groups, the use of VC is a preferred method, with people stating that, ' $I$ was very satisfied' [OAP], 'I much preferred video' and 'I don't think I would have gone to my appointment if it had to leave the house' [AP]. It's 'so much better using technology' [AP] as "it

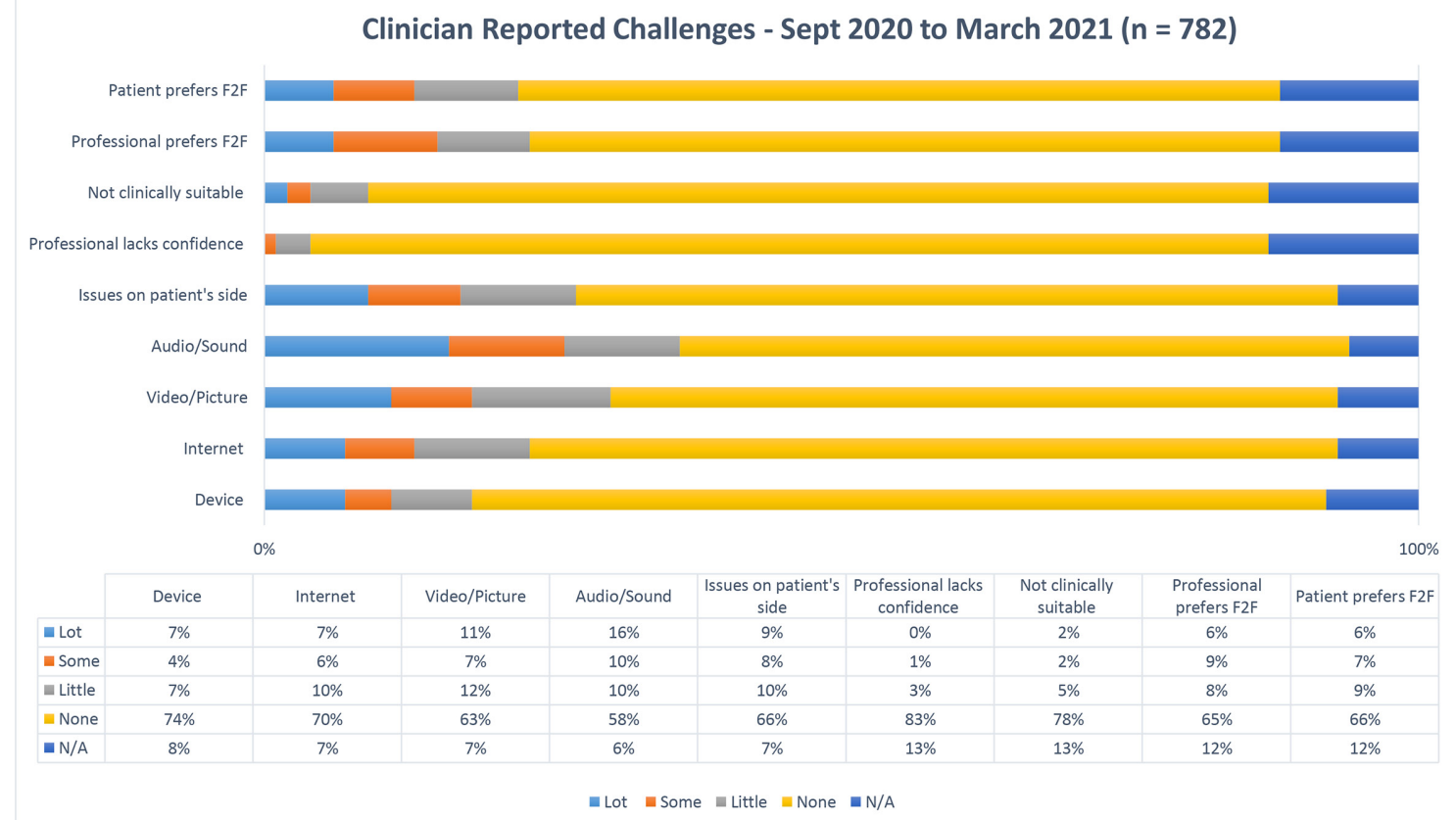

Figure 7 Distribution of clinician challenges using VC. N/A, non-applicable; VC, video consulting. 
was conducted in the safety of my home which is my protection bubble... this is an excellent way for my therapy sessions' [OAP].

An interesting relationship between preference of $\mathrm{VC}$ use and the perception of value is commonly influenced by the measure of $\mathrm{VC}$ benefits and its challenges. For example, it is frequently reported by patients on the 'ease' of use, stating that VC is 'much easier to use than I had anticipated' $(O A P)$, and 'I feel like it's better on video call as there are so many benefits and it's super easy to use' [AP]. The benefit of 'convenience' for mental health patients in particular, was rated highly, generally due to 'not having to leave the house' [CP] or 'easier, due to disability' [AP]. The benefits of 'savings of time and money' is commonly reported, in that 'no travel time or parking issues' [OAP, CP] and 'It seems obvious that this would save NHS and patients a lot of time' [AP]. Furthermore, 'flexibility' is considered a significant benefit to patients because '... the flexibility of the VC, suits my lifestyle perfectly' [AP].

In addition to these primary benefits are the positive impacts they have on clinical outcomes such as reduced 'stress and anxiety' on the patient. An AP states, 'It's much easier for a veteran (with PTSD) not to attend, or stress about finding parking space or waiting with strangers in a waiting room' [AP]. Another patient reports that 'having a VC is so much easier for me. I have epilepsy and suffer from stress seizures too, so travelling is always a risk—this is much better' [AP]. Positive impacts on other patient outcomes were also reported, such as the ability to 'share feelings better [via VC]' and the ability to 'talk freely [AP], yet still being able to 'build up a relationship' with the clinician [AP], and 'support us as a family' [AP, CP]. It was also noted by older age adult patients that ' $V C$ was more relaxing than attending a clinical appointment' (OAP) and 'more helpful than face to face sessions I can open up more' (OAP).

In addition, patients report how VC enables 'independent care', by 'managing my own symptoms' [OAP], being provided with virtual 'strategies for self-care' and providing 'really good insight'. One older adult patient states that 'It was easy to talk and describe my feelings and emotions using the video call and I was able to understand more about my condition' $(O A P)$. Similarly, a parent of a CAMHS patient says that it 'gives significant insight to patient symptoms and behaviours... a more agreeable and clearer understanding' $[\mathrm{CP}]$.

From the perspective of the mental health clinicians, compared with telephone calls, VC provides additional clinical flexibility, such as the use of 'visual aids', and increases the range of available observational data for assessment of mental state, physical health and mood. It also provides the opportunity to communicate visually through body language, eye contact and facial expressions, and is 'able to make a good connection with the patient, able to pick up non-verbal body language and facial expressions. 'Video quality and sound were both good' [AC] and 'clinically, I am really pleased with the way the emotions of my client was able to be shared' [CC].

Despite the many benefits, it is not uncommon to see patients report technological restraints such as 'unstable internet'; 'audio stalling'; 'glitchy video' or 'frozen screen' and at times, device incompatibilities such as the inability to 'turn the camera front facing' [CP, AP]. For clinicians, the restraints tend to be more specific such as, 'I don't like position of webcam on top of desktop pc screen - hard to look at patient on screen and camera at same time' [CC] or that it is 'difficult to be as clinically effective due to the lag in communication' and 'not being able to pick up on the interpersonal nuances' [AC]. And for others, they find ways around the difficulties, such as 'due to technical issues with sound we had to move to a phone call on but we kept the visual going' [CC].

It is likely that we will need to continue to gather data to analyse whether communicating via $\mathrm{VC}$ is as good as face-toface for all mental health treatments and population groups and to see what, if any, therapeutic impact this has on assessment and treatment, particularly where additional reasonable adjustments for communication are required or to accommodate the needs of groups such as younger children who communicate through play rather than speech.

Now that the technical infrastructure is improving and many patients and clinicians have both increased their skills and confidence in the use of $\mathrm{VC}$, the potential for offering the choice of routine and sustained use of $\mathrm{VC}$ in mainstream mental health provision looks very promising, where clinically appropriate. Some adult mental health patients state 'this really is the future' [AP], how 'it's a real life changer' [AP], and for some 'I want all appointments this way' [AP], ' $V C$ 's are an absolute godsend for me... I find attending face-toface stressful and unpleasant... in my own home it's more beneficial because I feel more relaxed' [AP]. Others have said they'd prefer a 'blended approach' suggesting that 'maybe a mix of the two' [AP, OAP] and 'I'd be happy for some VC, but I need face-to-face for processing' [AP]. 'I would always prefer face-to-face when possible... but I am happy to continue with a video call' [AP].

\section{DISCUSSION}

VC has been in existence for many years, and has an emerging evidence base for its use in healthcare, including mental health. However, it was not embraced as a form of mainstream NHS healthcare delivery in the UK until the COVID-19 precipitated sudden and large-scale change. This change has been out of necessity rather than choice. Traditionally preferring face-to-face methods, often with scepticism among clinicians that VC cannot replace face-to-face contact, particularly for encounters which require high levels of engagement such as therapy, or visual observation of body language and micro-expressions, such as first assessments. ${ }^{15}$

This mixed-methods study using both surveys and interviews is, to our knowledge, the most in-depth national evaluation of the use, value, benefits and challenges of VC for mental health remote appointments in the UK, from both patient and clinician perspectives. Our data suggest that VC has a highlevel of satisfaction and acceptability to both patients and clinicians, but also that patients embrace $\mathrm{VC}$ 
more than clinicians. It also suggests that for many patients, VC is a preferred option to face-to-face contact. However, it is important to acknowledge that this is not the general consensus, and that due to the pandemic we may be experiencing a temporary NHS appreciation bias, where there is gratitude for receiving a service at all or that $\mathrm{VC}$ preference is being driven out of a temporary risk of infection. Our findings suggest that overall, despite voluntary participation in the feedback surveys and interviews which may have introduced some bias and missed representation, VC clearly demonstrates in the evidence that we have available to us, as well accepted within mental health services and, by all age groups among CAMHS, adult and OAP. Further research and evaluation is needed to explore this in the long-term, including which mental health interventions and conditions are best suited to VC, perhaps looking specifically at VC, blended, or face to face only approaches and how mental health provision, commissioning, training and practice will evolve as a result. Considering how VC also impacts on socio-cultural factors, health inequalities, digital poverty and the need for reasonable adjustments are also important research areas moving forward. It will also be important to continue to measure key metrics such as equity of access, miles travelled, patient outcomes and experience measures for both patients and clinicians across these approaches to compare their quality over time.

The advances due to VC constitute a shift in how NHS mental healthcare services function; this is a transition away from a long-established traditional face-to-face care paradigm which has often placed the onus on patients to attend appointments at the healthcare provider's premises. This shift is also in line with governmental aspirations to increase the use of digital technology and accessibility of healthcare services. ${ }^{16-18}$ As the opportunity to return to face-toface work emerges, it will be important to encourage a culture of open, transparent and joint decision making between the patient and clinician about the clinically appropriate choice of $\mathrm{VC}$, blended or face to face, rather than returning to services where faceto-face appointments are the expected and default norm. Mental health services have a unique opportunity postpandemic to offer increased patient choice, reflected by need and preference, where flexibility of mode of delivery is seen as a core part of care planning.

\section{LIMITATIONS}

There are many strengths to this study, including its mixed-methodology approach, and its relatively large and representative sample for Wales. However, the study did have some limitations. For example, the small sample of OAP impacted on the group being analysed separately in survey 1 and the small sample of CAMHS data in survey 2. There were also limits on available participant demographic data, which provides limited context within the results. Furthermore, due to the voluntary and anonymised nature of the survey data collection, it is unclear as to how often recurring participants completed the survey, thus potentially skewing the sample.

\section{CONCLUSION}

This article presents real data gathered from operation of a national VC service, concerning use, value, benefits and challenges encountered by the patients and clinicians in mental health services in Wales. There are cost savings associated with utilising VC, and it could be particularly useful for those patients who find it difficult to leave their homes (eg, severe anxiety, caring responsibilities), have long distances to travel to see clinicians face to face, or simply feel more comfortable with VC. As we emerge from the COVID-19 pandemic and services begin to go back to normal, it would be important to retain $\mathrm{VC}$ as an alternative to face-to-face appointments, allowing for patients and clinicians to be able to choose their modality according to personal preference and clinical suitability.

\section{Twitter Gemma Johns @teccymru and Oliver John @olliejon}

Contributors GJ contributed to the main design of the study and development of the research questions, the main structure and write-up of the paper, and final amendments to the manuscript. JW, BW and GJ analysed the data, with $A A, S K$ and $M O$ supervision. All authors discussed and interpreted the data once analysed and helped structure the manuscript. AB, JT, $0 \mathrm{~J}$ and $A A$ contributed to the clinical understanding of the findings and shaped the discussion, conclusions and recommendations. AA was responsible for overseeing the full development of the study design and data collection, the analysis and development and final sign-off of manuscript from a clinical and programme perspective. All authors contributed to proof-reading and amendments of the final manuscript.

Funding Technology Enabled Care (TEC) Cymru and its NHS Wales Video Consulting Service is funded by the Welsh Government (no award number provided).

Competing interests None declared.

Patient consent for publication Consent obtained directly from patient(s).

Ethics approval This was initially obtained from their host Health Board Aneurin Bevan University Health Board Research \& Development Department (Reference Number: SA/1114/20), and then national approval was obtained from all other Health Boards in Wales.

Provenance and peer review Not commissioned; externally peer reviewed.

Data availability statement Data are available on reasonable request. All data relevant to the study are included in the article or uploaded as online supplemental information. All analysed data is published on the TEC Cymru website in the format of a full report of all data for the public to view. To access this reports please see https://digitalhealth.wales/tec-cymru. Other data can be requested as a reasonable request to the corresponding author.

Supplemental material This content has been supplied by the author(s). It has not been vetted by BMJ Publishing Group Limited (BMJ) and may not have been peer-reviewed. Any opinions or recommendations discussed are solely those of the author(s) and are not endorsed by BMJ. BMJ disclaims all liability and responsibility arising from any reliance placed on the content. Where the content includes any translated material, BMJ does not warrant the accuracy and reliability of the translations (including but not limited to local regulations, clinical guidelines, 
terminology, drug names and drug dosages), and is not responsible for any error and/or omissions arising from translation and adaptation or otherwise.

Open access This is an open access article distributed in accordance with the Creative Commons Attribution Non Commercial (CC BY-NC 4.0) license, which permits others to distribute, remix, adapt, build upon this work non-commercially, and license their derivative works on different terms, provided the original work is properly cited, appropriate credit is given, any changes made indicated, and the use is non-commercial. See: http://creativecommons.org/licenses/by-nc/4.0/.

ORCID iDs

Gemma Johns http://orcid.org/0000-0001-9823-4822

Alka Ahuja http://orcid.org/0000-0003-2658-2021

\section{REFERENCES}

1 Zhou X, Snoswell CL, Harding LE, et al. The role of telehealth in reducing the mental health burden from COVID-19. Telemed J $E$ Health 2020;26:377-9 https://www.liebertpub.com/doi/full/

2 Whaibeh $\mathrm{E}$, Mahmoud $\mathrm{H}$, Naal H. Telemental health in the context of a pandemic: the COVID-19 experience. Curr Treat Options Psychiatry 2020;7:198-202 https://link.springer.com/article/

3 Greenhalgh T, Wherton J, Shaw S, et al. Video consultations for covid-19. BMJ 2020;368:m998 https://www.bmj.com/content/368/ bmj.m998

4 Donaghy E, Atherton H, Hammersley V, et al. Acceptability, benefits, and challenges of video consulting: a qualitative study in primary care. Br J Gen Pract 2019;69:e586-94 https://bjgp.org/content/69/ 686/e586.short

5 Adams S, Rice M, Jones S. Reimbursement, and interstate practice. J Am Psychiatr Nurses Assoc 2018;24:295-305 https://journals. sagepub.com/doi/abs/

6 Waugh M, Voyles D, Thomas MR. Telepsychiatry: benefits and costs in a changing health-care environment. Int Rev Psychiatry 2015;27:558-68 https://www.tandfonline.com/doi/abs/

7 Langarizadeh M, Tabatabaei MS, Tavakol K, et al. Telemental health care, an effective alternative to conventional mental care: a systematic review. Acta Inform Med 2017;25:240 https://www.ncbi. nlm.nih.gov/pmc/articles/PMC5723163/
8 Smith AC, Thomas E, Snoswell CL, et al. Telehealth for global emergencies: implications for coronavirus disease 2019 (COVID-19). J Telemed Telecare 2020;26:309-13 https://journals.sagepub.com/ doi/full/

9 Gloff NE, LeNoue SR, Novins DK, et al. Telemental health for children and adolescents. Int Rev Psychiatry 2015;27:513-24 https://www. tandfonline.com/doi/abs/

10 Nelson E-L, Cain S, Sharp S. Considerations for conducting telemental health with children and adolescents. Child Adolesc Psychiatr Clin N Am 2017;26:77-91.

11 Mahmoud H, Vogt EL, Sers M, et al. Overcoming barriers to largerscale adoption of telepsychiatry. Psychiatr Ann 2019;49:82-8 https:// www.childpsych.theclinics.com/article/S1056-4993(16)30079-7/ abstract

12 Reinhardt I, Gouzoulis-Mayfrank E, Zielasek J. Use of telepsychiatry in emergency and crisis intervention: current evidence. Curr Psychiatry Rep 2019;21:63 https://link.springer.com/article/

13 Technology Enabled Care (TEC). Cymru. Available: https:// digitalhealth.wales/tec-cymru

14 The NHS video consulting service. Available: https://digitalhealth. wales/tec-cymru/vc-service

15 Courtney E, Blackburn D, Reuber M. Neurologists' perceptions of utilising tele-neurology to practice remotely during the COVID-19 pandemic. Patient Educ Couns 2021;104:452-9 https://www. sciencedirect.com/science/article/pii/S0738399120306911

16 Welsh Government. Health in Wales | document | informed health and care - a digital health and social care strategy for Wales, 2015. Wales.nhs. Available: http://www.wales.nhs.uk/document/280369/ info/ [Accessed 20 Apr 2021].

17 Welsh Government. Report: improving people's lives through digital technologies (Welsh Government, 2018) [Internet], 2018. Digitalcommunities.gov.wales. Available: https://www. digitalcommunities.gov.wales/report-improving-peoples-livesthrough-digital-technologies-welsh-government-2018/ [Accessed 20 Apr 2021].

18 UK Government. Making it work: harnessing the power of health information technology to improve care in England. Report of the National Advisory group on health information technology in England, 2016. Available: https://assets.publishing.service.gov.uk/ government/uploads/system/uploads/attachment_data/file/550866/ Wachter Review Accessible.pdf 